\title{
COMMENTARY
}

\section{METTL3-Mediated m6A Modification Links Liver Homeostasis and Pathology}

Wenbo Ma and Tong Wu

From the Department of Pathology and Laboratory Medicine, Tulane University School of Medicine, New Orleans, Louisiana

RNA modification is a new paradigm of epigenetic regulation of gene expression. This evolving field has been coined as $R N A$ epigenetics or epitranscriptomics. Extensive studies over recent years have shown that RNA modifications affect multiple steps of RNA metabolism, including RNA export, splicing, processing, stability, degradation, and translation. Among different forms of RNA modifications, N6-methyladenosine (m6A) modification is the most prevalent form of messenger RNA modification in eukaryotes, which is critically implicated in the regulation of cellular functions. ${ }^{1}$

m6A modification of RNA is catalyzed by methyltransferase consisting predominantly of methyltransferaselike 3 (METTL3) that serves as the methyltransferase core to provide the methyltransferase activity, with METTL14 playing an essential structural role to facilitate catalysis. The m6A-modified RNA is recognized by reader proteins which act by recognizing and binding to $\mathrm{m} 6 \mathrm{~A}$ to regulate RNA functions. Recent transcriptome-wide m6A mapping and mechanistic investigations have unveiled specific mRNAs that are regulated by $\mathrm{m} 6 \mathrm{~A}$ which provide mechanistic links connecting $\mathrm{m} 6 \mathrm{~A}$ to various cellular processes.

m6A in RNA is an epigenetic modification in which a hydrogen atom $(-\mathrm{H})$ connected to the sixth nitrogen atom (N6) on adenine is replaced by a methyl group (-CH3). With the advent of high-throughput sequencing technology, many m6A RNA modification sites have been discovered, which are mainly concentrated in exons and $3^{\prime}$ untranslated regions (UTRs), with the highest concentration near the termination codon. Each transcript contains three to five or more m6A modification sites, accounting for $0.1 \%$ to $0.4 \%$ of total adenine (m6A/A). In eukaryotic RNAs, m6A mostly occurs in the critically conserved motif RRACH $(R=G, A$, and $\mathrm{U} ; \mathrm{H}=\mathrm{U}, \mathrm{A}$, and $\mathrm{C}$ ) and while it is most common in mRNA, it is also widely found in long non-coding RNA (lncRNA), tRNA, rRNA, and microRNA (miRNA).
Pertinent to the topic of liver pathobiology, recent studies have shown that m6A RNA modification plays important roles in several aspects of liver biology and liver diseases, from lipid metabolism, liver regeneration, to nonalcoholic fatty liver disease (NAFLD), viral hepatitis, and carcinogenesis. The readers are referred to a recent Review article which highlights some of the recognized roles of $\mathrm{m} 6 \mathrm{~A}$ modification in liver diseases. ${ }^{2}$ Until now, the functional impact and mechanism of METTL3-mediated m6A modification and RNA epigenetics in liver pathobiology are far from clarified. In particular, how METTL3-mediated m6A RNA modification regulates liver physiological processes in the absence of exogenous injury remains largely unknown.

In this issue of The American Journal of Pathology, Barajas et $\mathrm{al}^{3}$ offer new insight into the role of METTL3mediated m6A RNA modification in liver homeostasis. The authors generated liver-specific METTL3 knockout (KO) mice (M3LKO) by crossing Mettl3 ${ }^{\mathrm{fl} / \mathrm{fl}}$ mice with Alb-Cre mice and their findings from these $\mathrm{KO}$ mice demonstrate an important role of METTL3 in the maintenance of liver homeostasis. As detailed in their article, the authors show that depletion of METTL3 in hepatocytes leads to reduction of m6A modification of polyadenylated RNAs along with several molecular and histopathological alterations. Alterations included dysregulation of genes involved in metabolism, cell cycle control, and circadian rhythms. The M3LKO livers exhibited pathological features associated with NAFLD (such as microvesicular steatosis, hepatocyte

Supported by the NIH grants CA219541, CA102325 and CA226281.

Accepted for publication October 26, 2021.

Disclosures: None declared.

Address correspondence to Tong Wu, M.D., Ph.D., Department of Pathology and Laboratory Medicine, Tulane University School of Medicine, 1430 Tulane Ave., SL-79, New Orleans, LA 70112. E-mail: twu@tulane. edu 
ballooning, ductular reaction, pleomorphic nuclei, DNA damage, foci of altered hepatocytes, focal lobular and portal inflammation, and elevated serum ALT/ALP levels). However, it is worth noting that these changes are neither unique nor specific for NAFLD, as they may also be present in other liver disease conditions. Intriguingly, the Mettl3depleted hepatocytes were highly proliferative, with decreased numbers of binucleate hepatocytes and increased nuclear polyploidy. Another interesting aspect of the study is that the M3LKO livers showed reduced expression of several key metabolic transcripts regulated by circadian rhythm and the protein levels of core clock transcription factors (including BMAL1 and CLOCK) were significantly decreased. The decrease in total Bmall and Clock mRNAs along with increase in their nuclear levels in M3LKO livers suggested impaired nuclear export mediated by m6A modification of Bmall and Clock mRNAs. Together, their results establish METTL3-mediated m6A RNA modification as an important regulator of liver homeostasis and function.

Prior to the study by Barajas et $\mathrm{al}^{3}{ }^{3}$ two independent groups had described the role of METTL3 in NAFLD by using mice with liver specific knockout of METTL3 ${ }^{4,5}$ Xie et $\mathrm{al}^{4}$ reported that METTL3 deletion in adult hepatocytes (using AAV8-TBG-Cre in Mettl3fl/fl mice) under high fat diet feeding led to improved insulin sensitivity and reduced fatty acid synthesis with decreased expression of fatty acid synthase. A separate study by $\mathrm{Li}$ et $\mathrm{al}^{5}$ reported that hepatocyte-specific knockout of METTL3 (using albuminCre in Mettl3fl/fl mice) ameliorated high fat diet-induced metabolic disorders (as reflected by slowing weight gain, reducing lipid accumulation, and improving insulin sensitivity). The above two independent studies both point toward the role of METTL3-mediated m6A RNA modification in NAFLD.

Two additional studies analyzed the role of METTL3- or METTL14-mediated m6A RNA modification in liver regeneration ${ }^{6,7}$ Meng et $\mathrm{al}^{6}$ generated mice with liverspecific knockout of METTL3 (using the albumin-Cre/ loxp system) and analyzed the role of METTL3 in liver regeneration following partial hepatectomy. The authors observed that deletion of METTL3 in hepatocytes delayed liver regeneration. Cao et $\mathrm{al}^{7}$ generated mice with hepatocyte-specific deletion of METTL3 or METTL14 (also using the albumin-Cre/loxp system) and compared the roles of METTL3 and METTL14 in liver regeneration after partial hepatectomy. Cao et al observed that METTL14, but not METTL3, depletion impaired liver regeneration under their system. Thus, the exact roles of METTL3 versus METTL14 in m6A RNA modification and their implications in liver regeneration remain to be further defined. Further studies are needed to determine whether METTL3-METTL14 heterodimer is required for optimal liver regeneration and function.

Whereas the existing studies have focused primarily on the role of METTL3-mediated m6A RNA modification in liver disease models, the role of METTL3 in liver physiological processes remains largely unexplored. The experimental results presented by Barajas et $\mathrm{al}^{3}$ show that METTL3 plays an important role in regulating liver polyploidy and circadian rhythm, which are essential for maintaining liver homeostasis.

Polyploidy of hepatocytes is an important phenomenon during liver development. This process creates genetic diversity and is also beneficial for adult liver recovery from injury. Additionally, polyploidization can cause genomic instability and can be associated with certain pathological conditions, including impairment of liver regeneration and hepatic carcinogenesis. ${ }^{8}$ Thus far, whether METTL3 is implicated in the regulation of cell polyploidy has not been described. The study by Barajas et $\mathrm{al}^{3}$ provides novel evidence that the level of METTL3 expression influences cell polyploidy in mouse liver. As detailed in their article, depletion of METTL3 led to upregulated expression of genes involved in cell cycle control with decreased binucleation and increased nuclear polyploidy. In addition to polyploidy change, hepatocytes from M3LKO mice were proliferative and showed signs of DNA damage. ${ }^{3}$ The presence of both apoptotic and proliferative hepatocytes in Mettl3-depleted livers suggested that hepatocytes enter cell cycle to compensate for the damaged cells, which become polyploid due to impaired cytokinesis. In other words, the authors speculate that depletion of METTL3 may cause hepatocyte death, which can lead to compensatory proliferation of surviving hepatocytes. Alternatively, it is also possible that the M3LKO hepatocytes might be inherently more replicative. The authors further describe that downregulation of WEE1 (a G2-M cell cycle regulator) may perturb hepatic cell cycle progression in M3LKO mice. Given the potential role of METTL3 in hepatocellular carcinoma (HCC), ${ }^{9}$ it remains to be determined whether and how the aforementioned cell cycle dysregulation, polyploidy, and proliferation observed with METTL3 deletion may play a role in HCC development.

Previous studies have demonstrated that m6A mRNA modification is involved in the regulation of the circadian clock in murine livers. ${ }^{10,11}$ For example, knockdown of METTL3 has been reported to lead to the elongation of the circadian period and a delay in RNA processing. ${ }^{10}$ A recent study shows that m6A mRNA modification oscillations in murine liver depend upon a functional circadian clock. ${ }^{11}$ Hepatic deletion of Bmal1, an important component of the circadian clock gene regulatory network, not only influences the METTL3 and YTHDF2 proteins but also alters mRNA methylation patterns in murine livers. ${ }^{11}$ These observations, along with other existing studies, suggest that m6A RNA modification is implicated in circadian clock regulation under physiological conditions. In this context, the study by Barajas et $\mathrm{al}^{3}$ provides additional evidence that circadian rhythm is controlled by METTL3-mediated m6A modification. The authors show that liver-specific deletion of METTL3 reduced the expression of several key metabolic 
transcripts regulated by circadian rhythm, and the protein levels of core clock transcription factors (including BMAL1 and CLOCK) were significantly decreased. It is plausible that decreased synthesis of BMAL1 and CLOCK proteins is likely due to inhibition of mRNA export, although the possibility of regulation at the level of translation, posttranslational modifications, and nuclear import of these proteins cannot be excluded. The study by Barajas et $\mathrm{al}^{3}$ demonstrates an important role of METLL3-mediated m6A RNA modification in the regulation of liver circadian rhythm. Given that hepatic polyploidy is known to be regulated by circadian rhythm, ${ }^{12}$ it is conceivable that METTL3-medidated m6A RNA modification may also influence hepatic polyploidy, in part, through regulation of circadian rhythm.

In summary, while RNA modifications are implicated in various liver disorders, little is known about their roles in hepatic physiological processes. The article by Barajas et $\mathrm{al}^{3}$ in this issue of The American Journal of Pathology provides new information indicating the role of METTL3 and m6A modification in regulation of liver homeostasis. Future studies are warranted to determine the function of RNA modifications in additional hepatic cell populations and to investigate other aspects of the liver epitranscriptomic regulatory network under both physiological and pathological conditions.

\section{References}

1. Zaccara S, Ries RJ, Jaffrey SR: Reading, writing and erasing mRNA methylation. Nat Rev Mol Cell Biol 2019, 20:608-624

2. Zhao Z, Meng J, Su R, Zhang J, Chen J, Ma X, Xia Q: Epitranscriptomics in liver disease: basic concepts and therapeutic potential. J Hepatol 2020, 73:664-679

3. Barajas JM, Lin CH, Sun HL, Alencastro F, Zhu AC, Aljuhani M, Navari L, Yilmaz SA, Yu L, Corps K, He C, Duncan AW, Ghoshal K:
METTL3 regulates liver homeostasis, hepatocyte ploidy, and circadian rhythm-controlled gene expression in mice. Am J Pathol 2022, 192 : $56-71$

4. Xie W, Ma LL, Xu YQ, Wang BH, Li SM: METTL3 inhibits hepatic insulin sensitivity via N6-methyladenosine modification of Fasn mRNA and promoting fatty acid metabolism. Biochem Biophys Res Commun 2019, 518:120-126

5. Li Y, Zhang Q, Cui G, Zhao F, Tian X, Sun BF, Yang Y, Li W: m(6)A regulates liver metabolic disorders and hepatogenous diabetes. Genomics Proteomics Bioinformatics 2020, 18:371-383

6. Meng J, Zhao Z, Xi Z, Xia Q: Liver-specific Mettl3 ablation delays liver regeneration in mice. Genes Dis 2020, [Epub ahead of print] doi: 10.1016/j.gendis.2020.11.002

7. Cao X, Shu Y, Chen Y, Xu Q, Guo G, Wu Z, Shao M, Zhou Y, Chen M, Gong Y, Li C, Shi Y, Bu H: Mettl14-mediated m6A modification facilitates liver regeneration by maintaining endoplasmic reticulum homeostasis. Cell Mol Gastroenterol Hepatol 2021, 12: 633-651

8. Donne R, Saroul-Ainama M, Cordier P, Celton-Morizur S, Desdouets C: Polyploidy in liver development, homeostasis and disease. Nat Rev Gastroenterol Hepatol 2020, 17:391-405

9. Chen MN, Wei L, Law CT, Tsang FHC, Shen JL, Cheng CLH, Tsang LH, Ho DWH, Chiu DKC, Lee JMF, Wong CCL, Ng IOL, Wong CM: RNA N6-methyladenosine methyltransferase-like 3 promotes liver cancer progression through YTHDF2-dependent posttranscriptional silencing of SOCS2. Hepatology 2018, 67: $2254-2270$

10. Fustin JM, Doi M, Yamaguchi Y, Hida H, Nishimura S, Yoshida M, Isagawa T, Morioka MS, Kakeya H, Manabe I, Okamura H: RNAmethylation-dependent RNA processing controls the speed of the circadian clock. Cell 2013, 155:793-806

11. Zhong X, Yu J, Frazier K, Weng X, Li Y, Cham CM, Dolan K, Zhu X, Hubert N, Tao Y, Lin F, Martinez-Guryn K, Huang Y, Wang T, Liu J, He C, Chang EB, Leone V: Circadian clock regulation of hepatic lipid metabolism by modulation of m(6)A mRNA methylation. Cell Rep 2018, 25:1816-1828.e4

12. Chao HW, Doi M, Fustin JM, Chen H, Murase K, Maeda Y, Hayashi H, Tanaka R, Sugawa M, Mizukuchi N, Yamaguchi Y, Yasunaga JI, Matsuoka M, Sakai M, Matsumoto M, Hamada S, Okamura H: Circadian clock regulates hepatic polyploidy by modulating Mkp1-Erk1/2 signaling pathway. Nat Commun 2017, 8:2238 